\title{
Is it time to reconsider combination lipid therapy in high-risk diabetic patients?
}

\author{
Alexander J. Blood ${ }^{\mathrm{a}}$ and Richard A. Krasuski ${ }^{\mathrm{b}}$
}

Diabetic patients are among the highest risk populations for atherosclerosis-related cardiovascular events [1] and LDL cholesterol (LDL-C) remains, the most effective target for pharmacological intervention in these patients. The American Diabetic Association recommends moderate-intensity statin therapy in addition to lifestyle modification for diabetic patients aged at least 40 or those less than 40 with additional cardiac risk factors, and high-intensity statin therapy for diabetic patients aged at least 40 with additional cardiac risk factors [2]. These guidelines do not currently recommend combination therapy due to the failures of prior trials examining add-on therapy, including the Action to Control Cardiovascular Risk in Diabetes (ACCORD) lipid trial [3]. Furthermore, clinicians are generally reluctant to prescribe multidrug regimens due to concerns about drug-drug interactions and the potential negative impact on patient compliance.

Despite the accepted benefits of lifestyle modification and statin therapy, the yearly cardiovascular event rate in diabetic patients remains upward of 3\% [4]. Although statins effectively lower LDL-C, they also upregulate proprotein convertase subtilisin/kexin type 9 (PCSK9) concentrations, which may lessen the extent of therapeutic benefit $\left[5,6,7^{*}\right]$. Circulating PCSK9 levels closely correlate with atherogenic dyslipidemia analyzed by NMR in diabetic patients [8"]. This provides further impetus to reconsider combination therapy in diabetic patients, particularly those at highest risk for clinical events, such as following an acute coronary syndrome.

Recent studies have shown that adding ezetimibe improves LDL-C levels in diabetic patients compared with simply increasing statin dose alone. Ezetimibe also improves lipoprotein subclass profile and lipoprotein-associated phospholipase A2 levels more than statin therapy alone [9"], as well as potentially offsetting statin-related elevation in plasma PCSK9 levels [10]. The recently published Improved Reduction of Outcomes: Vytorin Efficacy International Trial (IMPROVE-IT) demonstrated that ezetimibe and simvastatin were more successful than simvastatin alone in reducing LDL-C and decreasing cardiovascular events in patients following acute coronary syndrome [11"']. These differences become even more apparent in the subanalysis of diabetic patients [12]. Despite similar baseline lipid levels, diabetic patients on combination therapy experienced a $43 \mathrm{mg} / \mathrm{dl}$ decrease in LDL-C after 1 year compared with $23 \mathrm{mg} / \mathrm{dl}$ with simvastatin alone. This resulted in a $5.5 \%$ absolute risk reduction in the composite primary end point, highlighted by a $4.4 \%$ absolute risk reduction in myocardial infarction and a $2.7 \%$ absolute risk reduction in ischemic stroke.

The recent approval of PCSK9 inhibitors provides another tempting therapeutic option for the high-risk diabetic population on statins. Safety of PCSK9 inhibitors as add-on therapy to statins has been demonstrated and the magnitude of LDL-C reduction in published trials has been quite impressive [13"']. However, at present, these agents must be parenterally administered and are generally cost prohibitive and have yet to demonstrate a clear reduction in clinical events in the general population. Their role in diabetic dyslipidemia also remains to be defined.

The tide is clearly turning toward re-examining adjunctive therapy to statins in diabetic patients with high cardiovascular risk. It is expected that the continued development of well tolerated and effective agents will further facilitate this process.

\section{Acknowledgements}

None.

\section{Financial support and sponsorship}

None.

anternal Medicine and ${ }^{\mathrm{b}}$ Adult Congenital Heart Center, Duke University Medical Center, Durham, North Carolina, USA

Correspondence to Richard A. Krasuski, MD, Duke University Medical Center, 2301 Erwin Rd, Durham, NC 27710, USA. Tel: +1919684 2407; fax: +1 919681 7917; e-mail: richard.krasuski@duke.edu

Curr Opin Lipidol 2016, 27:310-311

DOI:10.1097/MOL.0000000000000306 


\section{Conflicts of interest}

R.A.K. is a consultant for Actelion and Bayer Pharmaceuticals and a scientific advisory board member for Ventripoint (unfunded). There are no conflicts of interest.

\section{REFERENCES AND RECOMMENDED READING}

Papers of particular interest, published within the annual period of review, have been highlighted as:

- of special interest

- of outstanding interest

1. Mozaffarian D, Benjamin EJ, Go AS, et al. Heart disease and stroke statistics2015 update: a report from the American Heart Association. Circulation 2015; $131: e 29-e 39$.

2. American Diabetes Association. 8. Cardiovascular disease and risk management. Diabetes Care 2016; 39 (Suppl 1):S60-S71.

3. The ACCORD Study Group. Ginsberg HN, Elam MB, Lovato LC, et al. Effects of combination lipid therapy in type 2 diabetes mellitus. N Engl J Med 2010; 362:1563-1574.

4. Cholesterol Treatment Trialists' (CTT) Collaborators. Kearney PM, Blackwell L, Collins R, et al. Efficacy of cholesterol lowering therapy in 18,686 people with diabetes in 14 randomised trials of statins: a meta-analysis. Lancet 2008; 371:117-125.

5. Awan Z, Seidah NG, MacFadyen F G, et al. Rosuvastatin, proprotein convertase subtilisin/kexin type 9 concentrations, and LDL cholesterol response: the JUPITER trial. Clin Chem 2012; 58:183-189.

6. Welder G, Zineh I, Pacanowski MA. High-dose atorvastatin causes a rapid sustained increased in human serum PCSK9 and disrupts its correlation with LDL cholesterol. J Lipid Res 2010; 51:2714-2721.

7. Li S, Jun Li J. PCSK9: A key factor modulating atherosclerosis. J Atheroscler - Thromb 2015; 22:221-230.

The review highlights the developing understanding of PCSK9 and its effects on lipid metabolism, glucose metabolism, and inflammation. By discussing the in-vitro and in-vivo experiments to date of PCSK9, it provides guidance for the clinical risks and opportunities that are likely to surface from PCSK9 safety and outcomes studies.
8. Guardiola M, Plana N, lbaretxe D, et al. Circulating PCSK9 levels are positive

- correlated with NMR-assessed atherogenic dyslipidemia in patients with high cardiovascular risk. Clin Sci 2015; 128:877-882.

Investigators studied detailed NMR lipid profiles of 267 diabetic and metabolic syndrome patients who were not receiving any lipid-lowering therapy and found strong correlations with PCSK9 levels and triglycerides, apolipoprotein B, total cholesterol, and other proatherogenic circulating lipoprotein particles.

9. Le N, Tomassini J, Tershakovec A, et al. Effect of switching from statin mono-

- therapy to ezetimibe/simvastatin combination therapy compared with other intensified lipid-lowering strategies on lipoprotein subclasses in diabetic patients with symptomatic cardiovascular disease. J Am Heart Assoc 2015; 4:1-12.

The substudy of diabetic patients from a randomized, double-blind study, involving 86 international centers compared the effects of different dosages and the addition of medications on lipid profiles. When treating with low-dose statin, the addition of ezetimibe compared with doubling the dose of statin resulted in significant decreases in LDL-C concentrations, increases in HDL cholesterol concentrations, and improved lipoprotein-associated phospholipase A2 activity.

10. Sahebkar A, Simental-Mendia LE, Guerrero-Romero F, et al. Effect of statin therapy on plasma proprotein convertase subtilisin kexin 9 (PCSK9) concentrations: a systematic review and meta-analysis of clinical trials. Diabetes Obes Metab 2015; 17:1042-1055.

11. Cannon $C P$, Blazing $M A$, Giugliano RP, et al. Ezetimibe added to statin therapy -1. after acute coronary syndromes. N Engl J Med 2015; 372:2387-2397.

The landmark IMPROVE-IT study was a randomized, double-blind trial adding ezetimibe to simvastatin compared with placebo and simvastatin in patients hospitalized for acute coronary syndrome. Patients who received ezetimibe plus simvastatin had lower LDL levels and a $2 \%$ absolute risk difference in the composite primary end point of cardiovascular death, nonfatal myocardial infarction, unstable angina requiring rehospitalization, coronary revascularization, or nonfatal stroke. There were 4933 diabetic patients among the 18144 patients. Among these patients, the absolute risk reduction in cardiovascular events was $5.5 \%$.

12. Giguliano RP, CP Cannon, MA Blazing, et al. for the IMPROVE-IT Investigators. Benefit of adding ezetimibe to statin therapy on cardiovascular outcomes and safety in patients with vs without diabetes: the IMPROVE-IT trial. Presented at ESC Congress 2015. London, England, UK. Abstract 1947

13. Chapman MJ, Stock JK, Ginsberg HN. PCSK9 inhibitors and cardiovascular

-1isease: heralding a new therapeutic era. Curr Opin Lipidol 2015; 26:511520.

An excellent and up-to-date review of the PCSK9 inhibitors, which reviews the biology of these agents and the studies that have been performed examining their safety and efficacy. The authors also provide a series of unanswered questions to stimulate more critical appraisal of this new drug class.

\section{FURTHER RECOMIMENDED READING}

Kernan WN, Viscoli CM, Furie KL, et al. Pioglitazone after ischemic stroke or - transient ischemic attack. N Engl J Med 2016. [Epub ahead of print] A randomized, double-blind trial comparing pioglitazone with placebo in patients with recent ischemic stroke or transient ischemic attack who had evidence of insulin resistance. The primary outcome of stroke or myocardial infarction occurred in $9.0 \%$ of patients in the pioglitazone group compared with $11.8 \%$ of patients in the placebo group (hazard ratio 0.76 .) The rate of progression of diabetes was also lower in the pioglitazone group (3.8\%) than placebo $(7.7 \%)$ with a hazard ratio of 0.48 . In the safety analysis, pioglitazone was associated with a higher incidence of bone fracture, weight gain, and edema. Notably, pioglitazone was not associated with heart failure, incident cancer, or death. The authors did not directly examine lipid effects in this study or whether they were the mechanism of risk reduction, though pioglitazone is known to have multiple favorable effects on diabetic dyslipidemia.

Tsujita K, Sugiyama S, Sumida H, et al. Impact of dual lipid-lowering strategy with

- ezetimibe and atorvastatin on coronary plaque regression in patients with percutaneous coronary intervention. J Am Coll Cardiol 2015; 66:495-507. The prospective, randomized, controlled, multicenter study randomly assigned 246 patients undergoing percutaneous coronary intervention to receive atorvastatin alone or atorvastatin plus ezetimibe daily. These patients had an index as well as a subsequent (after 9-12 months of treatment) coronary catheterization with intravascular ultrasound measurements. Patients in the dual therapy group showed superiority with regard to LDL-C lowering as well as with coronary artery plaque regression when compared with those on statin monotherapy. 